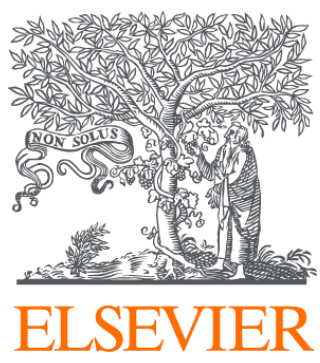

Since January 2020 Elsevier has created a COVID-19 resource centre with free information in English and Mandarin on the novel coronavirus COVID-

19. The COVID-19 resource centre is hosted on Elsevier Connect, the company's public news and information website.

Elsevier hereby grants permission to make all its COVID-19-related research that is available on the COVID-19 resource centre - including this research content - immediately available in PubMed Central and other publicly funded repositories, such as the WHO COVID database with rights for unrestricted research re-use and analyses in any form or by any means with acknowledgement of the original source. These permissions are granted for free by Elsevier for as long as the COVID-19 resource centre remains active. 


\title{
A robust high-throughput fluorescent polarization assay for the evaluation and screening of SARS-CoV-2 fusion inhibitors
}

\author{
Xinjian Yin ${ }^{a}$, Litong Chen ${ }^{a}$, Siwen Yuan ${ }^{a}$, Lan Liu ${ }^{\text {a,c }}$, Zhizeng Gao a,b,c, " \\ ${ }^{a}$ School of Marine Science, Sun Yat-sen University, Zhuhai 519080, China \\ ${ }^{\mathrm{b}}$ State Key Laboratory of Applied Optics, Changchun Institute of Optics, Fine Mechanics and Physics, Chinese Academy of Sciences, Changchun 130033, China \\ ${ }^{\mathrm{c}}$ Southern Marine Science and Engineering Guangdong Laboratory (Zhuhai), Zhuhai 519080, China
}

\section{A B S T R A C T}

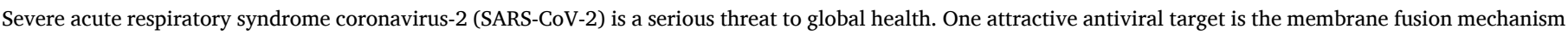

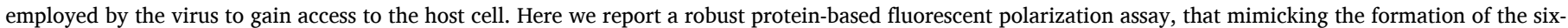

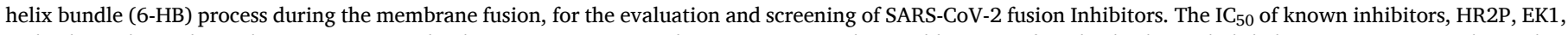

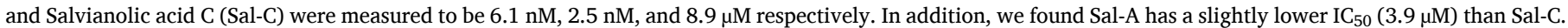

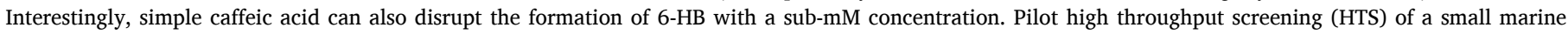

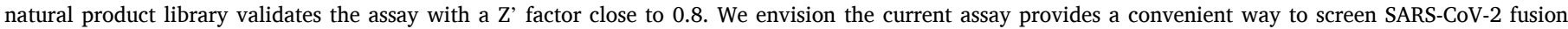
inhibitors and assess their binding affinity.

\section{Introduction}

As of Feb 2021, the severe acute respiratory syndrome coronavirus-2 (SARS-CoV-2) [1-2] has infected more than 100 million people and cause two million lives. Although several drugs have been "repurposed" for the treatment of SARS-CoV-2 infection, [3-4] there is still a pressing need to identify novel anti-SARS-CoV-2 therapeutics.

Both SARS-CoV-1 and SARS-CoV-2 virus use their surface homotrimeric spike protein (S protein) to enter the host cells (Fig. 1) [5]. The S protein encodes two subunits: S1 and S2. The receptor-binding domain (RBD) from the S1 subunit has a strong binding affinity with the cell surface receptor, angiotensin-converting enzyme II (ACE2), and is responsible for the initial attachment [5]. This binding event triggers a conformational change of S2 subunit and the previously buried hydrophobic fusion peptides are exposed and insert into the host cell membrane. Subsequently, two heptad repeats (HR1 and HR2) form a thermodynamically favorable six-helix bundle (6-HB) postfusion structure to fuse the viral and cellular membranes together [6].

The formation of 6-HB structure is a conserved fusion mechanism shared by many viruses besides coronavirus, including influenza virus, human immunodeficiency virus (HIV), and respiratory syncytial virus (hRSV) $[7,8]$. It has been shown that peptides derived from the heptad repeats region are potent fusion inhibitors, and such a peptide, Enfuvirtide, has been approved for HIV-1 treatment for almost 20 years [9].
The HR2 peptide of SARS-CoV-1 and SARS-CoV-2 are $100 \%$ conserved, and also displayed potent inhibition activities in cell-based assays. [10-13]. Recently, the Lu group reported that a novel HR2-derived peptide, EK1, can disrupt the protein-protein interaction (PPI) during the formation of 6-HB, and inhibit multiple human coronaviruses at sub$\mathrm{nM}$ concentration $[11,12]$. Thus, fusion inhibitors could potentially be developed as broad-spectrum anti-coronavirus therapeutics [14].

It is well-known that PPI is hard to be disrupted by conventional small molecules [15]. Natural products are privileged structures in the drug discovery process, and it might be easier to find PPI modulators from natural products [16]. Recently, the Liu group identified a potent anti-SARS-CoV-2 fusion inhibitor, Salvianolic acid C (Sal-C, IC $\mathrm{IC}_{50} 3.85$ $\mu \mathrm{M})$ from a natural product library using a cell-cell fusion assay mediated by S protein of SARS-CoV-2 [17]. Deconvolution of the activity by native-polyacrylamide gel electrophoresis ( $N$-PAGE) assay indeed suggested that Sal-C inhibits the formation of 6-HB in a dose-dependent manner.

Cell-based phenotypic screening assays can be expensive and the resulting activities sometimes can be hard to deconvolute. Thus, a robust in vitro high-throughput evaluation and screening platform for SARSCoV-2 fusion inhibitors is highly needed. Here we present our effort on the development of such a platform using fluorescence polarization (FP) techniques and apply it for the evaluation of the binding potency of HR2, EK1, and several Sal family natural products. We found Sal-A has

\footnotetext{
* Corresponding author.

E-mail address: gaozhizeng@mail.sysu.edu.cn (Z. Gao).
} 
A

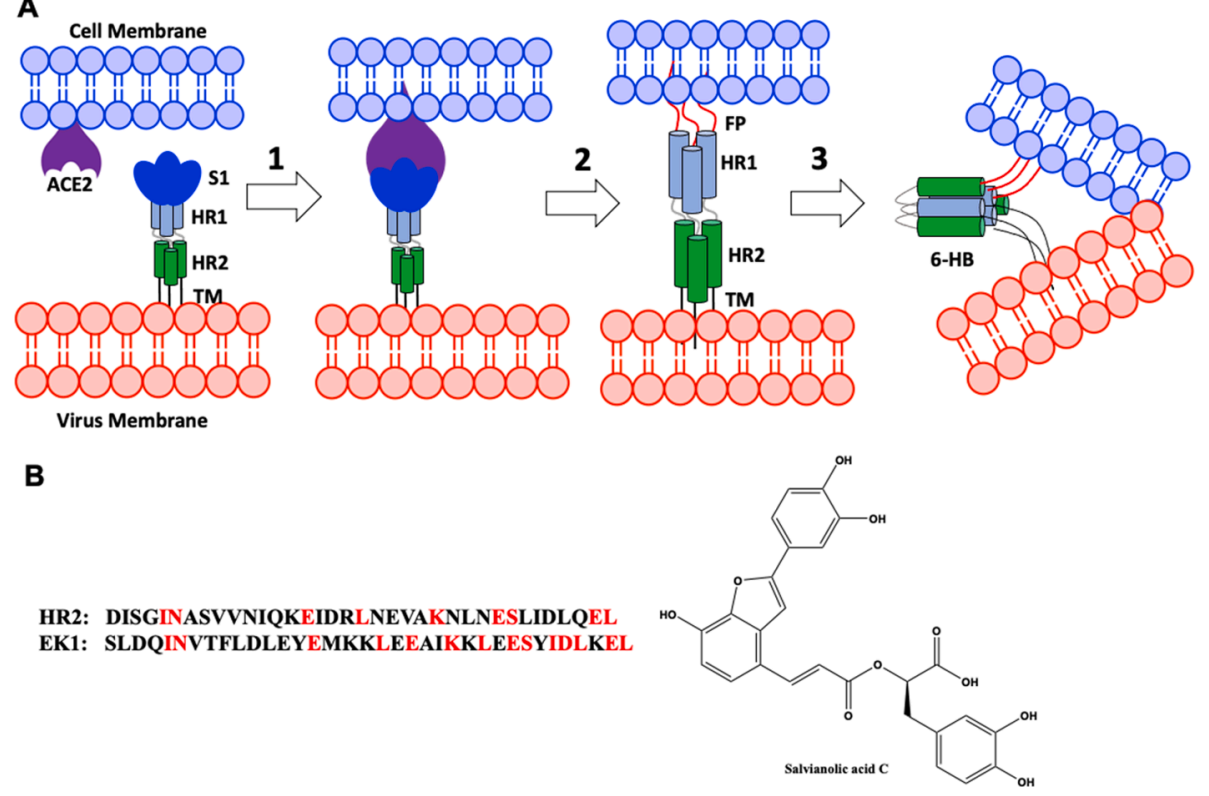

Fig. 1. A) SARS-CoV membrane fusion mechanism. Abbreviations: FP, fusion peptide; HR, heptad repeat; TM, transmembrane anchor. B) Known SARS-CoV-2 fusion inhibitors.

similar binding potency as Sal-C. In addition, we found that the precursor of natural product family of Sal which possesses a simple chemical structure as caffeic acid does, can inhibit the 6-HB formation with a $\mathrm{IC}_{50}$ of about $638 \mu \mathrm{M}$. Despite the relatively low potency, such a simple structure could potentially serve as a starting fragment for the fragment growing strategy [18] to increase the potency.

\section{Material and methods}

\subsection{Gene, plasmid, and materials}

The codon-optimized gene of 5-HB (the sequence was shown in Table S1) was synthesized by Tsingke Co., Ltd (Guangzhou, China) and inserted into plasmid pET-44b(+) and pET-28a $(+)$, respectively. The pCold-NusA plasmid was maintained in our laboratory.

The HR1P (CANQENSAIGKIQDSLSSTASALGKLQDVVNQNAQA LNTLVKQ) and HR2P (ELGDISGINASVVNIQKEIDRLNEVAKNLNES LIDLQELC) peptides were ordered from APeptide Co., Ltd (Shanghai, China). EK1 (SLDQINVTFLDLEYEMKKLEEAIKKLEESYIDLKEL) peptide was a gift from Prof. Lu Lu (School of Basic Medical Sciences, FudanJinbo Joint Research Center, Fudan University, Shanghai 200032, China). Salvianolic acid A, Salvianolic acid B, Lithospermic acid, Rosmarinic acid, and Caffeic acid were purchased from Aladdin Reagents Co. Ltd (Shanghai, China). Salvianolic acid C was purchased from MACKLIN Reagent Co. Ltd (Shanghai, China). All other chemicals and reagents were chemically pure and obtained commercially.

\subsection{Protein expression and purification}

The plasmids pET-44b-NusA-5HB, pET-28a-5HB, and pCold-NusA containing the gene encoding NusA-5HB, 5-HB and NusA (with $\mathrm{N}$-terminal His-tag) were transformed into competent $E$. coli BL21 (DE3) cells (Invitrogen) respectively. The recombinant $E$. coli was cultivated in LB medium with antibiotic (50 mg/L Kan for pET-28a-5HB; $100 \mathrm{mg} / \mathrm{L}$ Amp for pET-44b-NusA-5HB and pCold-NusA) at $37{ }^{\circ} \mathrm{C}$ and rotary shaking at $200 \mathrm{rpm}$. For protein expression, cells were induced with isopropyl- $\beta$-Dthiogalactopyranoside (IPTG) at a final concentration of $0.5 \mathrm{mM}$ until $\mathrm{OD}_{600}$ reached $0.6-0.8$, and then further cultured at $15{ }^{\circ} \mathrm{C}$ for $24 \mathrm{~h}$.

The collected cells were washed and resuspended in buffer A $(20 \mathrm{mM}$ sodium phosphate, $\mathrm{pH} 7.4$, containing $500 \mathrm{mM} \mathrm{NaCl}$ and $20 \mathrm{mM}$ imidazole). Resuspended cells were disrupted by ultrasonication in an ice bath, followed by centrifugation at $12000 \times \mathrm{g}$ for $30 \mathrm{~min}$ to remove cell debris. The supernatant was loaded onto a Ni-NTA column (Thermo Scientific, USA) pre-equilibrated with buffer A, and the proteins were eluted by an increasing gradient of imidazole (from 50 to $250 \mathrm{mM}$ ). The purities of the collected fractions were analyzed by SDS-PAGE. Fractions containing the pure target protein were gathered and then desalted by ultrafiltration. The purified proteins were concentrated and stored in $20 \%(\mathrm{v} / \mathrm{v})$ glycerol at $-80{ }^{\circ} \mathrm{C}$ until further use. The expression and purification of the proteins were analyzed by sodium dodecyl sulfate-polyacrylamide gel electrophoresis (SDS-PAGE, $4 \sim 20 \%$ ) (Figure S1). Protein concentrations were determined using a Bradford protein assay kit (Quick Start ${ }^{\mathrm{TM}}$, Bio-Rad, USA).

\subsection{HR2P-FL preparation}

Dissolving HR2P with $20 \mathrm{mM} \mathrm{pH} 7.5$ phosphate buffer (5 mM EDTA) to a concentration of $100 \mu \mathrm{M}$. A 25-fold molar excess of fluorescein-5maleimide $(2.5 \mathrm{mM})$ was added and then incubated at $25^{\circ} \mathrm{C}$ for $2 \mathrm{~h}$. After incubation, nonreacted fluorescein was removed by ultrafiltration using a $3 \mathrm{KDa}$ molecular-weight cutoff, and HR2P-FL was then purified by HPLC. The purified HR2P-FL was stored from light in single-use aliquots at $-20^{\circ} \mathrm{C}$.

\subsection{Fluorescence native polyacrylamide gel electrophoresis (N-PAGE)}

$10 \mu \mathrm{M}$ of HR2P-FL was incubated with $10 \mu \mathrm{M}$ of HR1P, 5-HB or NusA-5HB in pH $7.520 \mathrm{mM}$ PBS at $37{ }^{\circ} \mathrm{C}$ for $30 \mathrm{~min}$. After incubation, the sample was mixed with Tris-glycine native sample buffer (Invitrogen, Carlsbad, CA) at a ratio of 1:1 and was then loaded to $4 \% \sim 20 \%$ pre-cast gel ( $20 \mu \mathrm{L}$ each well). Gel electrophoresis was carried out with $100 \mathrm{~V}$ constant voltages at room temperature for $1.5 \mathrm{~h}$. The gel was imaged with a Tanon 1220 Gel Imaging System (Shanghai, China)。

\subsection{FP assays}

All reported concentrations represent the final assay conditions unless otherwise specified. Fluorescent polarization measurements were performed on a Spark ${ }^{\circledR}$ multimode microplate reader (Tecan, Switzerland), with an excitation filter at $485 \mathrm{~nm}$, and an emission filter 
at $535 \mathrm{~nm}$. The G-Factor was set at 1.06 (Note: The calibration is performed by measuring a $1 \mathrm{nM}$ fluorescein solution at room temperature and adjusting the G-factor to achieve a value of $27 \mathrm{mP}$; different microplate readers might need a different setting) [19]. Peptides were directly dissolved in water to make a $500 \mu \mathrm{M}$ stock solution and then stored at $-80^{\circ} \mathrm{C}$.

\subsubsection{Binding assays of HR2P-FL with NusA-5HB/5-HB}

Assays were performed manually in black 96 microplates (Cat. No. 3694, Corning, USA). Each reaction (100 $\mu \mathrm{L})$ contained $10 \mathrm{nM}$ of HR2PFL and $1 \mu \mathrm{M}$ of NusA-5HB, 5-HB or NusA in FP buffer (25 mM pH 7.5 PBS, $0.025 \%$ NP-40). The FP values were measured after $1 \mathrm{~h}$ incubation at $37{ }^{\circ} \mathrm{C} 200 \mathrm{rpm}$. All experiments were performed in duplicate.

\subsubsection{Saturation binding FP measurement}

Assays were performed manually in black 96 microplates (Cat. No. 3694, Corning, USA). Each well (100 $\mu \mathrm{L})$ contained 5 nM HR2P-FL and increasing concentrations $(0 \sim 128 \mathrm{nM})$ of NusA-5HB in FP buffer (25 $\mathrm{mM}$ pH 7.5 PBS, 0.025\% NP-40). The FP values were measured after $1 \mathrm{~h}$ incubation at $37{ }^{\circ} \mathrm{C} 200 \mathrm{rpm}$. The binding fraction $(f)$ of HR2P-FL with different NusA-5HB concentrations was calculated using the Eqs. (1) and (2).[20]. The obtained $f$ values were analyzed and plotted using GraphPad Prism 8 to calculate the binding dissociation constant $\left(K_{\mathrm{d}}\right)$ by fitting the experimental data using a one-site specific binding model. All experiments were performed in duplicate.

$A=\frac{2 P}{3-P}$

$f=\frac{A-A_{f}}{\left(A_{B}-A\right) Q+A-A_{F}}$

$P$ is the fluorescence polarization values, $A$ is the fluorescence anisotropy values; $A_{B}$ and $A_{F}$ denote fluorescence anisotropies of fully bound and free HR2P-FL, respectively; $Q$ is the ratio of fluorescence intensities of bound and free species measured under the same experimental conditions. In our assay, $Q$ is found to be close to 1 .

\subsubsection{Competitive FP assays of peptides}

Assays were performed manually in black 96 microplates (Cat. No. 3694, Corning, USA) contained $8 \mathrm{nM}$ NusA-5HB and increasing concentrations of peptides $(0,0.31,0.625,1.25,2.5,5,10,20,40,80,160$, $320 \mathrm{nM}$ ) in FP buffer (25 mM pH 7.5 PBS, 0.025\% NP-40) in a final volume of $90 \mu \mathrm{L}$. After $1 \mathrm{~h}$ incubation at $37{ }^{\circ} \mathrm{C} 200 \mathrm{rpm}, 10 \mu \mathrm{L}$ HR2P-FL $(5 \mathrm{nM})$ was added and additional $1 \mathrm{~h}$ incubation was followed at $37^{\circ} \mathrm{C}$ $200 \mathrm{rpm}$. The FP signal was measured. The binding fraction $(f)$ of HR2PFL with NusA-5HB at different concentrations of peptide was calculated using the Eqs. (1) and (2). The obtained $f$ values were plotted using GraphPad Prism 8 to calculate the $\mathrm{IC}_{50}$. All experiments were performed in duplicate.

\subsubsection{Competitive FP assays of small molecules}

Assays were performed manually in black 96 microplates (Cat. No. 3694, Corning, USA) contained $8 \mathrm{nM}$ NusA-5HB and increasing concentrations of small molecules $(0,0.001,0.01,0.1,1,10,100,1000 \mu \mathrm{M})$ in FP buffer (25 mM pH 7.5 PBS, 0.025\% NP-40, 4\% DMSO) in a final volume of $90 \mu \mathrm{L}$. After $1 \mathrm{~h}$ incubation at $37{ }^{\circ} \mathrm{C} 200 \mathrm{rpm}, 10 \mu \mathrm{L}$ of HR2PFL (5 nM) was added and additional $1 \mathrm{~h}$ incubation was followed at $37{ }^{\circ} \mathrm{C} 200 \mathrm{rpm}$. In order to eliminate the fluorescence interference of small molecules, fluorescence intensities parallel $\left(I_{=}\right)$, and perpendicular $\left(I_{\perp}\right)$ to the plane of exciting light were measured after incubation and the real FP values were calculated using the Eq. (3). The binding fractions $(f)$ of HR2P-FL with NusA-5HB at different concentrations of small molecules were calculated using the Eqs. (1) and (2). The obtained $f$ values were plotted using GraphPad Prism 8 to calculate the $\mathrm{IC}_{50}$. All experiments were performed in duplicate.

$P=\frac{\left(I_{=}-I_{=^{*}}\right)-G^{*}\left(I_{\perp}-I_{\perp *}\right)}{\left(I_{=}-I_{=^{*}}\right)-G^{*}\left(I_{\perp}-I_{\perp *}\right)}$

$I_{=*}$ and $I_{\perp} *$ are fluorescence intensities of same concentration small molecules parallel, and perpendicular to the plane of exciting light. $G$ is the $G$-Factor ( $1 \mathrm{nM}$ fluorescein solution at room temperature and adjusting the $\mathrm{G}$-factor to achieve a value of $27 \mathrm{mP}$ ).

\subsection{Molecular docking}

The solved post-fusion core of SARS-CoV-2 6-HB was selected as the docking structure (PDB code: 6LXT) [11]. In this structure, one of the HR2 chains was removed and the channel occupied by this HR2 was defined as the docking grid. Ligands were prepared using MGLTools 1.5.4 and docking was performed with auto-dock vina. The molecular docking poses were ranked according to calculated binding affinity and the top one was selected for further analysis. PyMOL (Version 1.5, Schro dinger, LLC) was used to prepare figures of the complexes.

\subsection{Screening the marine natural product library}

The marine natural product library $(10 \mathrm{mM}$ dissolved in $100 \%$ DMSO) in 96-well format was maintained in our laboratory. A daughter library (100 times dilution) was created by transferring $1 \mu \mathrm{L}$ of the stocks into $99 \mu \mathrm{L}$ of $40 \%$ DMSO. To black 96 -well microtiter plates were added $80 \mu \mathrm{L}$ of a premix containing $8 \mathrm{nM}$ NusA-5HB, buffer $(25 \mathrm{mM} \mathrm{pH} 7.5$
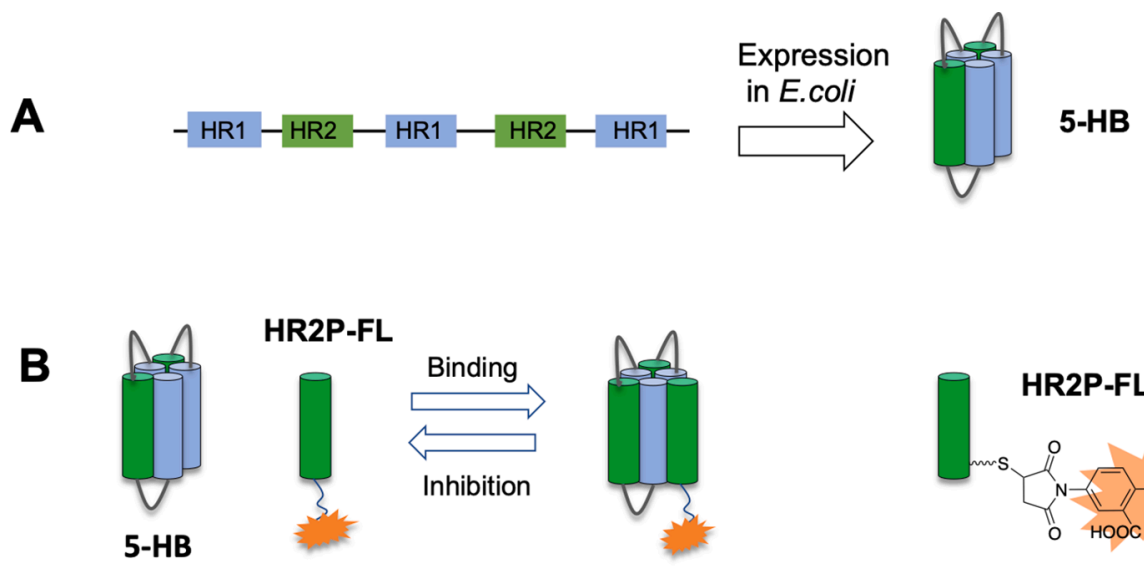

HR2P-FL

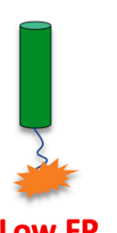

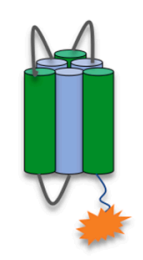

High FP

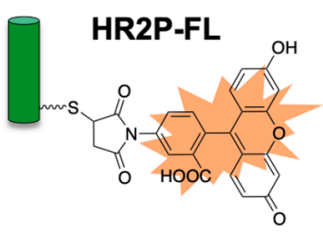

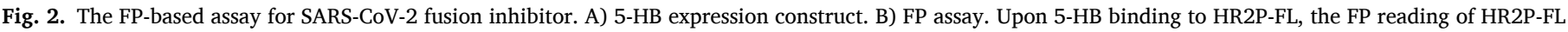
increases. If inhibitors can disrupt the binding between 5-HB and HR2P-FL, the FP reading decreases. 
A

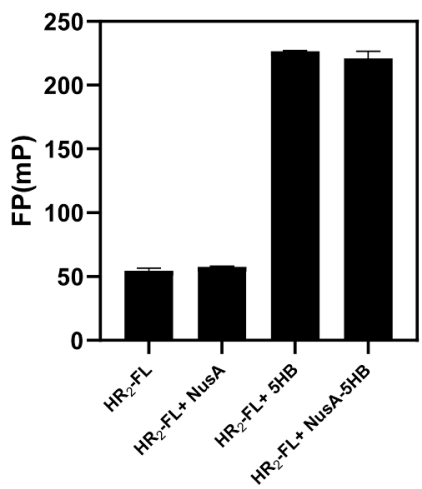

B

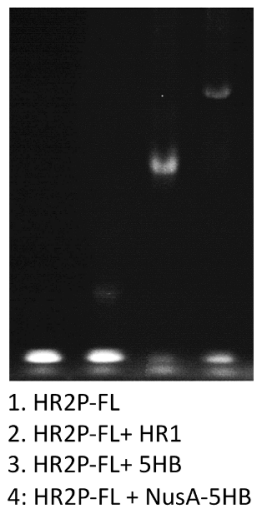

C

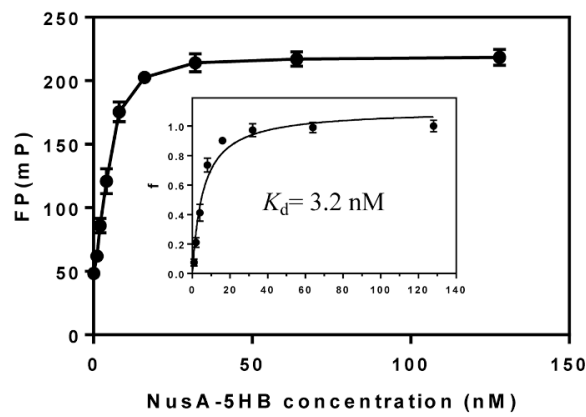

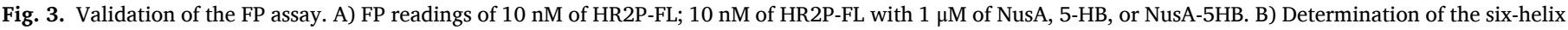

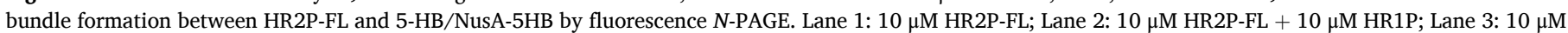

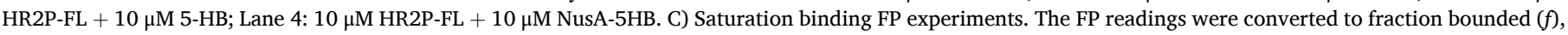
and then plotted against the NusA-5HB concentration to get $K_{\mathrm{d}}=3.2 \mathrm{nM}$. All experiments were performed in duplicate.

PBS, $0.025 \%$ NP-40). $10 \mu \mathrm{L}$ daughter library solution was added (10 $\mu \mathrm{M})$ and incubated at $37{ }^{\circ} \mathrm{C}$ for $1 \mathrm{~h}$. Then, $10 \mu \mathrm{L}$ of HR2P-FL $(5 \mathrm{nM})$ was added and additional $1 \mathrm{~h}$ incubation was followed at $37^{\circ} \mathrm{C} 200 \mathrm{rpm}$. FP signal was measured after incubation. (Note: All concentrations represent the final assay conditions).

For quality assessment of the FP assay, positive and negative controls were set in the same 96-well microtiter plates and subjected to identical conditions described in the above screening procedure. Positive controls refer to the reactions in the absence of inhibitors while negative controls refer to the reactions in the absence of NusA-5HB. Z' score was calculated according to the equation as follows [21]:

$Z^{\prime}=1-\frac{3\left(\sigma_{p}+\sigma_{n}\right)}{\left|\mu_{p}-\mu_{n}\right|}$

The $\sigma_{p}$ and $\sigma_{p}$ are the standard deviations of the FP signals of positive and negative controls; $\mu_{p}$ and $\mu_{n}$ are the mean of FP signals of positive and negative controls.

\section{Results}

\subsection{FP-based assays for fusion inhibitors.}

FP is a powerful technique for the investigation of ligand-receptor interactions in homogenous solutions and has been widely used in the drug discovery process $[19,22]$. The basic concept is to link a fluorophore to the ligand and the fluorophore-ligand conjugate has a low molecular weight, thus freely rotating in the solution to give a low FP signal. Upon the addition of the receptor (typical an enzyme with higher molecular weight), the binding between receptor-ligand would noncovalently link the fluorophore to a large object, and the rotation is restricted to give a high FP signal.

The Kim group previously design a small protein, 5-HB, by connecting the five of the 6-HB of HIV-1 with short linkers [23]. The resulting 5-HB has a strong binding affinity with the HR2 region of HIV1 and displaced a strong fusion inhibition activity. Subsequently, such a 5-HB construct was used in an in vitro FP assay for screening HIV fusion inhibitors [24]. Inspired by those results, we developed an FP-based assay for SARS-CoV-2 fusion inhibitors as shown in Fig. 2. An expression plasmid with short linkers to link three HR1 and two HR2 of SARSCoV-2 was constructed. The resulting 5-HB construct would be expected to have a high binding affinity with fluorescence-labeled HR2 peptides (HR2P) to increase the FP signal. An extra cysteine is included in the synthetic HR2P (there is no cysteine in the native sequence) to facilitate the labeling with fluorescein-5-maleimide to get HR2P-FL.

We found 5-HB is mostly expressed in $E$. coli as inclusion body and the yield of soluble 5-HB fraction is very low. We envision that a solubilization protein tag would aid the expression. Here we chose the E. coli-derived NusA tag (55 kDa) [25-26] as it has high intrinsic solubility and its large size can potentially increase the FP signal. Indeed, NusA tag has been employed to help the expression of HIV-5HB [27]. We are glad to found that NusA-5HB construct gave a very high expression yield, and about $30 \mathrm{mg}-50 \mathrm{mg} / \mathrm{L}$ of soluble NusA-5HB can be obtained after purification.

\subsection{Validation of the FP-based assays}

With the HR2P-FL, NusA-5HB, and 5-HB in hand, we first mixed 10 $\mathrm{nM}$ of HR2P-FL and excess of NusA-5HB and 5HB $(1 \mu \mathrm{M})$. After incubation for $1 \mathrm{~h}$, the FP reading was measured by a plate reader. HR2P-FL gave a relatively low FP reading of $50 \mathrm{mP}$, while NusA-5HB and 5-HB raise the FP reading to 220 and $230 \mathrm{mP}$, respectively (Fig. 3A). Although NusA-5HB (100 kDa) is significantly larger than 5-HB (35 $\mathrm{kDa}$ ), comparable FP readings were obtained. This may be because the internal linker between NusA and 5-HB is flexible and the NusA can not restrict the rotation of 5-HB [28-29]. To rule out that NusA itself would bind to HR2P-FL to increase the FP reading, we also expressed the NusA protein, and we found NusA did not change the FP reading, suggested NusA does not bind to HR2P-FL.

To further validate the binding between HR2P-FL and 5-HB, we performed native-polyacrylamide gel electrophoresis ( $N$-PAGE) analysis [12]. As shown in Fig. 3B, we found HR2P-FL and HR1P only formed a small amount of 6-HB, while HR2P-FL can form 6-HB effectively with either 5-HB and NusA-5HB. Since both experiments suggest HR2P-FL could bind to NusA-5HB to form NusA-6HB, we subsequently focused on the assay optimization with NusA-5HB.

\subsection{Saturation binding FP experiments.}

To develop a successful FP-based assay, the $K_{\mathrm{d}}$ between fluorescent ligand and receptor need to be determined by saturation binding experiment $[20,30]$. The concentrations of fluorescent ligand and receptor in the subsequence competitive FP assay need to be adjusted according to the saturation binding experiment: the fluorescent ligand concentration needs to be less than $2 K_{\mathrm{d}}$ to avoid stoichiometric titration; while the concentration of receptor needs to be adjusted so that the initial fraction of fluorescent ligand bound to receptor $\left(f_{0}\right)$ is between 0.5 and 0.8 . 


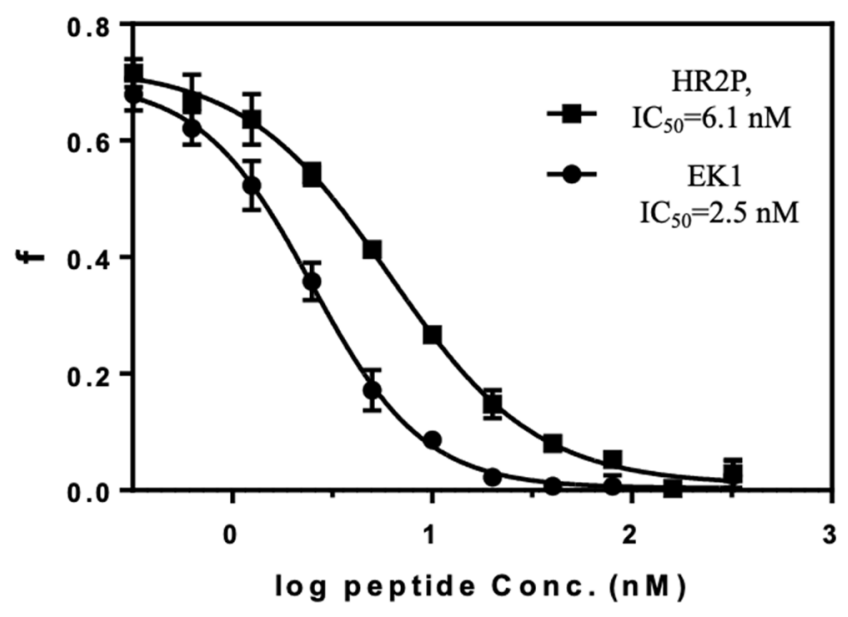

Fig. 4. Competitive FP assays with HR2P and EK1. The FP readings were converted to fraction bounded ( $f$ ), and then plotted against the HR2P and EK1 concentration to get $\mathrm{IC}_{50}=6.1 \mathrm{nM}$ for HR2P and $\mathrm{IC}_{50}=2.5 \mathrm{nM}$ for EK1, respectively. All experiments were performed in duplicate.

To determine the binding constant between NusA-5HB and HR2P-FL, increasing concentrations of NusA-5HB was added to the solution containing $5 \mathrm{nM}$ of HR2P-FL, and after incubate for $1 \mathrm{~h}$, FP signals were measured ((Fig. 3C)). As FP signals are not linear with the binding strength, [20] the anisotropy data were used to calculate the fraction bounded ratio $(f)$, which were then plotted against the NusA-5HB concentrations to get the $K_{\mathrm{d}}=3.2 \mathrm{nM}$.

\subsection{Competitive FP assays with HR2P and EK1}

With the saturation binding experiment data in hand, we set the
HR2P-FL and NusA-5HB concentrations to be $5 \mathrm{nM}$ (less than $2 K_{\mathrm{d}}$ ) and $8 \mathrm{nM}\left(f_{0}\right.$ is about 0.7$)$ respectively in the competitive FP assays. As shown in Fig. 4, IC 50 of HR2P and EK1 is 6.1 and $2.5 \mathrm{nM}$ respectively, suggesting that EK1 has a slightly higher binding toward 5-HB. However, it has been shown that HR2P has better activity than EK1 in cellbased pseudovirus infection assay $(0.98 \mu \mathrm{M}$ vs $2.38 \mu \mathrm{M})$ [12]. It is possible that the cell-based assay does not reflect the true binding affinity as peptides might have different cell-penetration abilities. Indeed, the Lu group recently reported that cholesterol or palmitic acid conjugated EK1 have much better in vivo activity than EK1, and the $\mathrm{IC}_{50}$ of both labeled peptides are in nM range [11].

\subsection{Evaluation of Sal family natural products with FP assays}

The Liu group reported that the nonpeptic natural product Sal-C is a potent SARS-CoV-2 fusion inhibitor with a $\mathrm{IC}_{50}$ of $3.85 \mu \mathrm{M}$ with an ACE2-expressing HEK293T cell, and a $\mathrm{EC}_{50}$ of $3.41 \mu \mathrm{M}$ with native SARS-CoV-2 virus [17]. The mode of action of Sal-C was elucidated by $N$ PAGE, and it was observed that Sal-C inhibits the formation of 6-HB between HR1P and HR2P in a dose-dependent manner. However, the binding affinity between Sal-C and SARS-CoV-S2, SARS-CoV-2-S2 (405 $\mu \mathrm{M}$ and $284.3 \mu \mathrm{M}$ respectively) is relatively low, possibly because S2 unit is not in the prefusion state. Our current assay has the potential to evaluate the true binding strength between Sal-C and spike protein in its prefusion state. In a competitive bind experiment, we obtained the $\mathrm{IC}_{50}$ of Sal-C to be $8.9 \mu \mathrm{M}$ (Fig. 5), comparable to that determined in the cellbased assay, suggest that Sal C possibly interacts at the interface of $5 \mathrm{HB}$ and HR2P. This result strongly suggested that our assay can be used to evaluate the potency of SARS-CoV-2 fusion inhibitors.

With these encouraging results in hand, we tested several Sal-C analogs that are commercially available: Sal-A, Sal-B, lithospermic acid, and rosmarinic acid (Fig. 5). The $\mathrm{IC}_{50}$ of Sal-A is about $3.9 \mu \mathrm{M}$, slightly better than Sal-C, suggesting the furan ring of Sal-C is not necessary. The
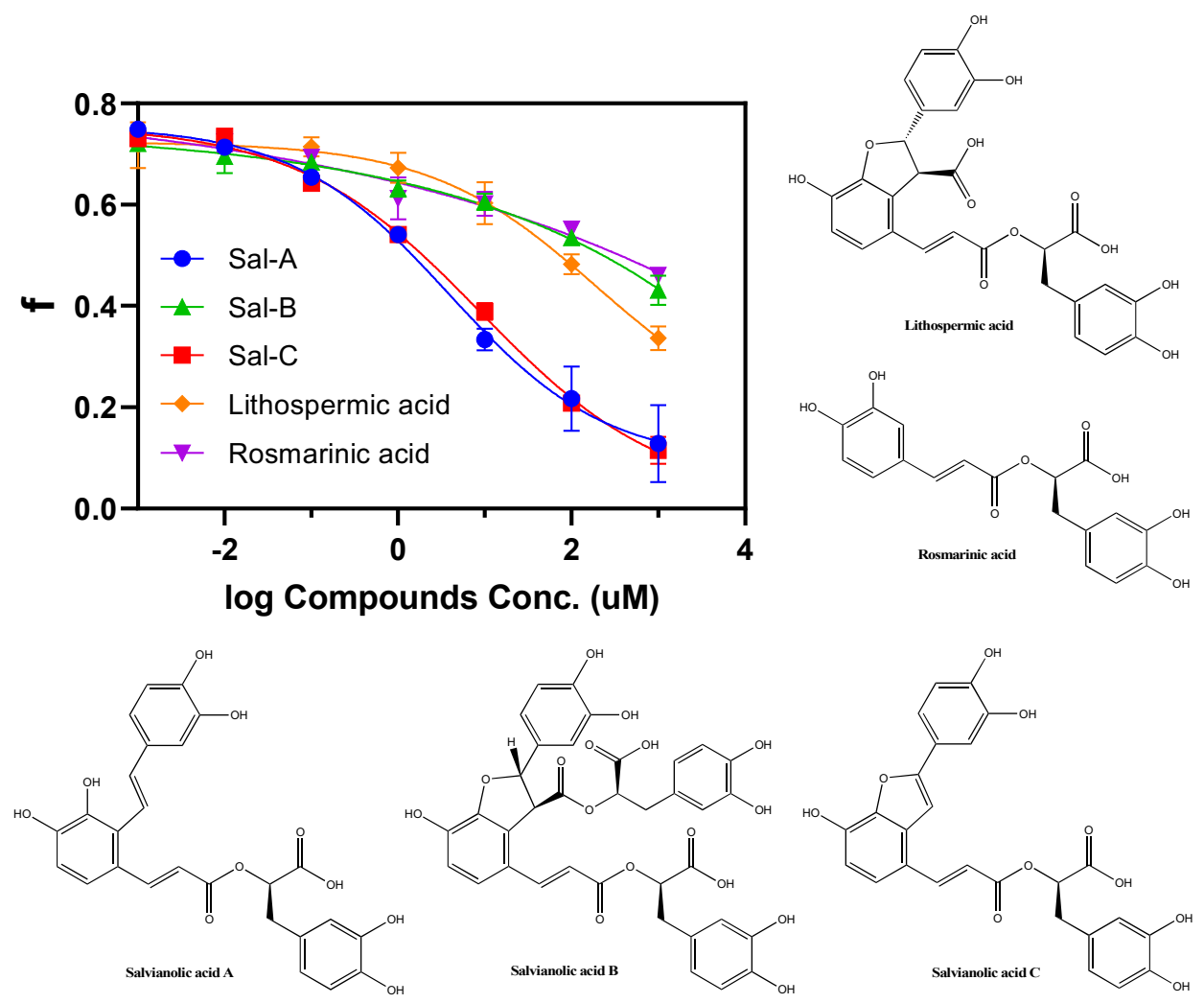

Fig. 5. Evaluation of Sal family natural products. The FP readings were converted to fraction bounded ( $f$ ), and then plotted against the respective natural product concentrations. All experiments were performed in duplicate. 

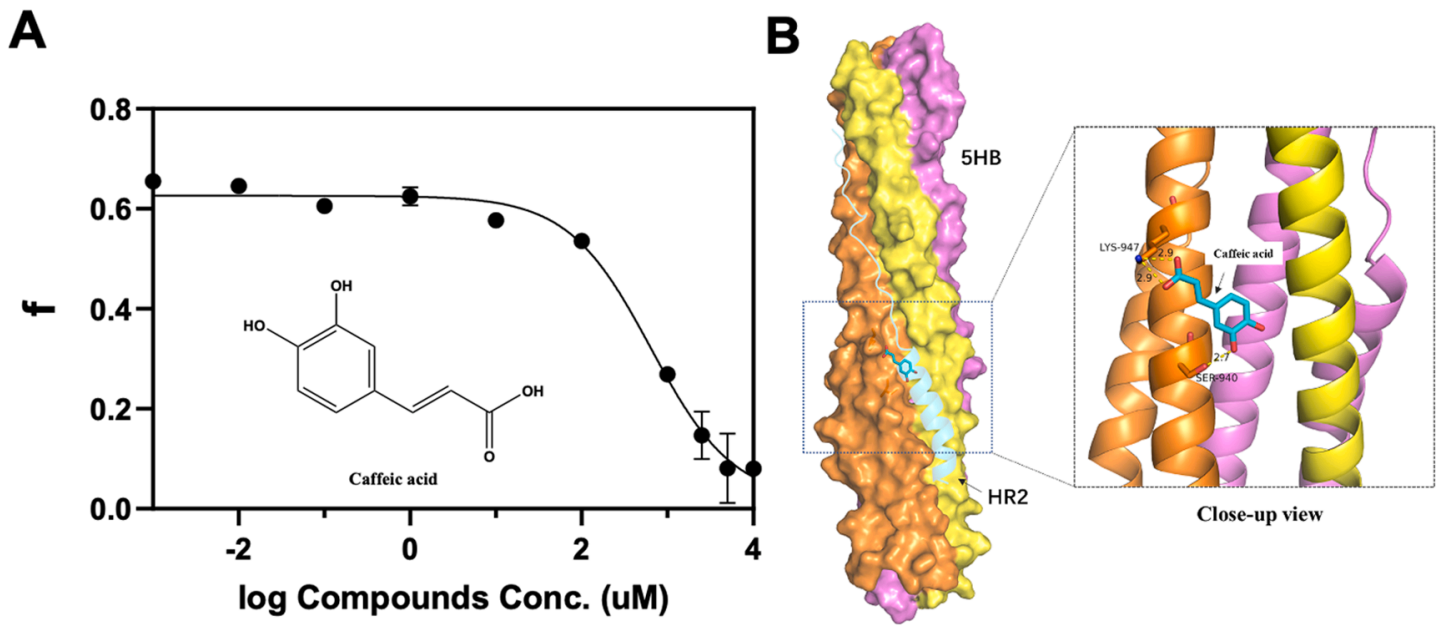

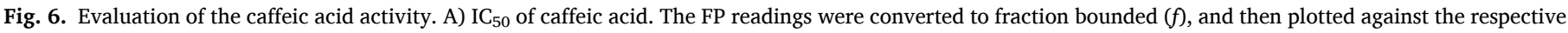

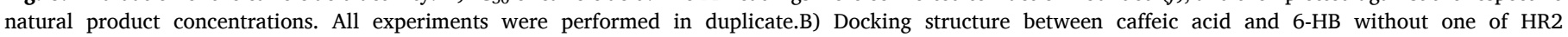
(PDB code:6LXT).
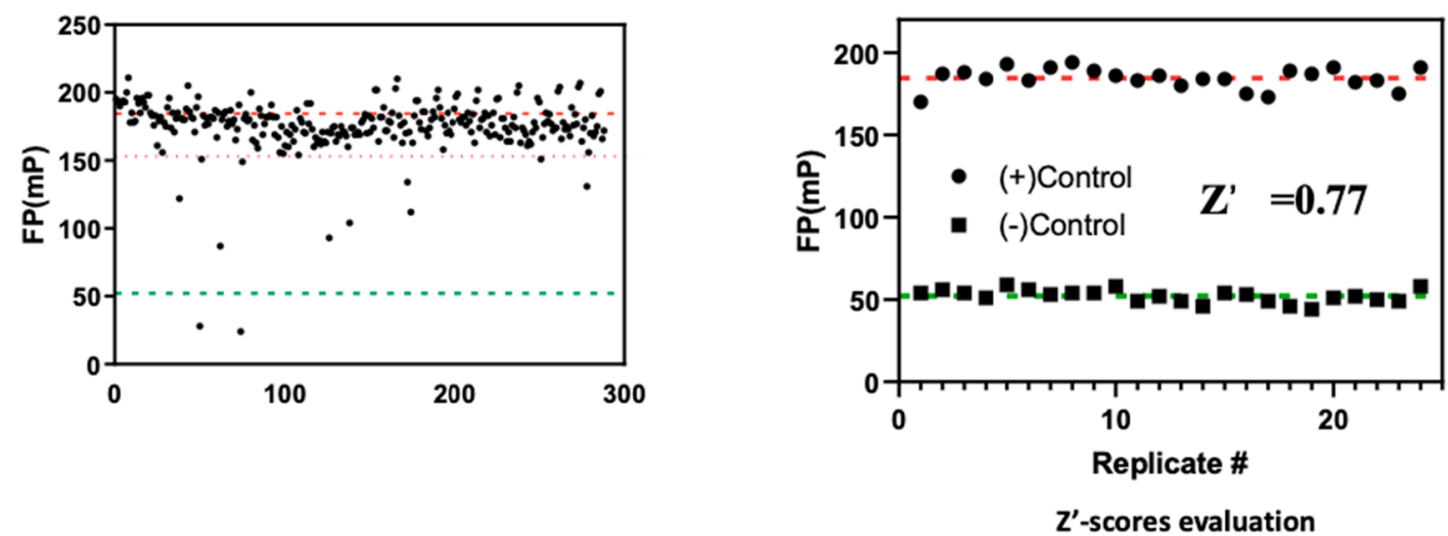

Fig. 7. Evaluation of the FP assay in HTS formats. A) Pilot HTS of marine natural product library. B) Z' scores evaluation.

other three natural products all have very week activities, and because of solubility issues, the $\mathrm{IC}_{50}$ values can not be obtained. Those results suggest although the furan ring is not necessary, the double bond of the furan ring is crucial for the activities.

Since Sal family natural products are oligomers of caffeic acid, we are curious that caffeic acid can also bind to 5-HB. As caffeic acid is much more soluble, we successfully obtained a IC I0 $_{5}$ of $638 \mu \mathrm{M}$ (Fig. 6A). PPI does not have a binding pocket, and usually it is hard to find binders with simple structures. It is pretty remarkable a simple structure such as caffeic acid could disrupt the interaction between 5-HB and HR2P in less than $\mathrm{mM}$ concentration. We then performed docking studies with the solved 6-HB crystal structure (PDB code:6LXT) [11], and it suggests that the carboxyl group of caffeic acid forms hydrogen bonding with Lys947, and para phenolic OH might interact with Ser940 (Fig. 6B). We envision that caffeic acid could potentially serve as a starting fragment in fragment-based drug discovery to increase the binding with 5-HB.

\subsection{High-throughput screening (HTS) of marine natural product library.}

As a marine natural product research group, we have a long-term interest to find bioactive molecules from the marine environment, and indeed we have previously successfully identified several potent inhibitors from marine natural product libraries with FP-based assays [31-32]. Therefore, we performed a pilot HTS with a small marine natural product library (about 300 compounds) at $10 \mu \mathrm{M}$ (Fig. 7A). Unfortunately, we did not find any active natural products from this small library after ruling out the fluorescent interfering compounds. However, we found the assay itself is very robust with a $\mathrm{Z}$ ' at 0.77 (Fig. 7B), which suggested that this assay can be applied to screen largesize commercial compound libraries.

\section{Discussion and conclusions}

SARS-CoV-2 continues to be a big threat to public health after the initial outbreak a year ago. The membrane fusion process employed by SARS-CoV-2 to enter the host cell is a highly conserved mechanism shared by many different types of viruses, suggesting that it is an attractive target to develop broad-spectrum antivirals.

Currently, research mainly relies on cell-based phenotypic assays to screen and evaluate the SARS-CoV-2 fusion inhibitors. Cell-based assays have advantages such as cell permeability factor already included, however, an economical, robust, and high-throughput in vitro assay is also an important complementary tool for screening and evaluation of binding potency of novel fusion inhibitors, and the further structure-activity relationship studies. In this study, we developed an in vitro assay to evaluate the inhibition of $6-\mathrm{HB}$ by determining the binding between 5-HB and fluorescently labeled HR2P with FP techniques.

FP-based assays typically required a large amount of protein, and we solved the 5-HB production problem by fusing it to a highly soluble NusA tag, and the resulting NusA-5HB construct can be produced in large quantities in $E$. coli (30-50 mg/L). In competitive FP experiments, the IC $\mathrm{I}_{50}$ of HR2P and EK1 peptides were determined to be 6 and $2.5 \mathrm{nM}$ 
respectively. Five Sal family natural products were evaluated with our assay, and we found Sal-A has a comparable activity with Sal-C, while the other three analogs have much lower activities. Interestingly, the simple monomer, caffeic acid also has a sub-mM activity, thus could potentially serval as a starting fragment for "fragment-based drug design".

Although our attempt to find fusion inhibitors from a small marine natural product library is not successful, the assay itself did demonstrate robustness with a Z' factor close to 0.8 in HTS formats. We envision that this assay could be applied to screen large commercial compound libraries as the production of large quantities of NusA-5HB and HR2P-FL is very straightforward. Another potential application of this assay is to screen fragment library as our assay can pick up sub-mM week activities, and the resulting fragment can be further optimized to increase the binding affinity with the aid of this assay.

\section{Declaration of Competing Interest}

The authors declare that they have no known competing financial interests or personal relationships that could have appeared to influence the work reported in this paper.

\section{Acknowledgements}

This study was supported by The National Key R\&D Program of China (2019YFC0312501), Guangdong Marine Economy Promotion Fund (Grant GDOE[2019]A21), the Open Fund of State Key Laboratory of Applied Optics (SKLAO2020001A10), Emergency Study on Prevention and Control of COVID-19 Epidemic (ZH22036302200036PWC). We thank Prof. Lu Lu for providing the peptide EK1.

\section{Appendix A. Supplementary material}

Supplementary data to this article can be found online at https://doi. org/10.1016/j.bioorg.2021.105362.

\section{References}

[1] P. Zhou, X.-L. Yang, X.-G. Wang, et al., A pneumonia outbreak associated with a new coronavirus of probable bat origin, Nature 579 (2020) 270.

[2] A.E. Gorbalenya, S.C. Baker, R.S. Baric, et al., The species Severe acute respiratory syndrome-related coronavirus: classifying $2019-\mathrm{nCoV}$ and naming it SARS-CoV-2, Nat. Microbiol. 5 (2020) 536.

[3] A. Sahebnasagh, R. Avan, F. Saghafi, et al., Pharmacological treatments of COVID 19, Pharmacol Rep 72 (2020) 1446.

[4] M.L. Holshue, C. DeBolt, S. Lindquist, et al., First Case of 2019 Novel Coronavirus in the United States, N Engl J Med 382 (2020) 929.

[5] D. Wrapp, N. Wang, K.S. Corbett, et al., Cryo-EM structure of the 2019-nCoV spike in the prefusion conformation, Science 367 (2020) 1260.

[6] S. Xia, L. Yan, W. Xu, et al., A pan-coronavirus fusion inhibitor targeting the HR1 domain of human coronavirus spike, Sci Adv 5 (2019) eaav4580.

[7] S. Martens, H.T. McMahon, Mechanisms of membrane fusion: disparate players and common principles, Nat. Rev. Mol. Cell Biol. 9 (2008) 543.

[8] B. Podbilewicz, Virus and cell fusion mechanisms, Annu. Rev. Cell Dev. Biol. 30 (2014) 111.
[9] T. Matthews, M. Salgo, M. Greenberg, et al., Enfuvirtide: the first therapy to inhibit the entry of HIV-1 into host CD4 lymphocytes, Nat. Rev. Drug. Discovery. 3 (2004) 215.

[10] V.K. Outlaw, F.T. Bovier, M.C. Mears, et al., Inhibition of coronavirus entry in vitro and ex vivo by a lipid-conjugated peptide derived from the sars-cov-2 spike glycoprotein hrc domain, Mbio 11 (2020), e01935.

[11] S. Xia, M. Liu, C. Wang, et al., Inhibition of SARS-CoV-2 (previously 2019-nCoV) infection by a highly potent pan-coronavirus fusion inhibitor targeting its spike protein that harbors a high capacity to mediate membrane fusion, Cell Res 30 (2020) 343.

[12] S. Xia, Y. Zhu, M. Liu, et al., Fusion mechanism of 2019-nCoV and fusion inhibitors targeting HR1 domain in spike protein, Cell Mol Immunol 17 (2020) 765.

[13] Y. Zhu, D. Yu, H. Yan, et al., Design of potent membrane fusion inhibitors against SARS-CoV-2, an emerging coronavirus with high fusogenic activity, J. Virol. 94 (2020).

[14] X. Wang, S. Xia, Q. Wang, et al., Broad-spectrum coronavirus fusion inhibitors to combat COVID-19 and other emerging coronavirus diseases, Int. J. Mol. Sci. 21 (2020) 3843.

[15] H. Lu, Q. Zhou, J. He, et al., Recent advances in the development of protein-protein interactions modulators: mechanisms and clinical trials, Signal Transduct Target Ther 5 (2020) 213.

[16] A.G. Atanasov, S.B. Zotchev, V.M. Dirsch, et al., Natural products in drug discovery: advances and opportunities, Nat. Rev. Drug Discovery 1 (2021).

[17] C. Yang, X. Pan, X. Xu, et al., Salvianolic acid C potently inhibits SARS-CoV-2 infection by blocking the formation of six-helix bundle core of spike protein, Signal Transduction and Targeted Therapy 5 (2020) 1.

[18] L. Hoffer, Y.V. Voitovich, B. Raux, et al., Integrated strategy for lead optimization based on fragment growing: the diversity-oriented-target-focused-synthesis approach, J. Med. Chem. 61 (2018) 5719.

[19] M.D. Hall, A. Yasgar, T. Peryea, et al., Fluorescence polarization assays in highthroughput screening and drug discovery: a review, Method. Appl. Fluoresc. 4 (2016), 022001.

[20] M.H. Roehrl, J.Y. Wang, G. Wagner, A general framework for development and data analysis of competitive high-throughput screens for small-molecule inhibitors of protein - protein interactions by fluorescence polarization, Biochemistry 43 (2004) 16056.

[21] J.-H. Zhang, T.D. Chung, K.R. Oldenburg, A simple statistical parameter for use in evaluation and validation of high throughput screening assays, J. Biomol. Screen. 4 (1999) 67.

[22] A. Uri, O.E. Nonga, What is the current value of fluorescence polarization assays in small molecule screening? Expert Opin. Drug Discov. 15 (2020) 131.

[23] M.J. Root, M.S. Kay, P.S. Kim, Protein design of an HIV-1 entry inhibitor, Science 291 (2001) 884.

[24] G. Frey, S. Rits-Volloch, X.-Q. Zhang, et al., Small molecules that bind the inner core of gp41 and inhibit HIV envelope-mediated fusion, Proc. Natl. Acad. Sci. 103 (2006) 13938.

[25] V. De Marco, G. Stier, S. Blandin, et al., The solubility and stability of recombinant proteins are increased by their fusion to NusA, Biochem. Biophys. Res. Commun. 322 (2004) 766.

[26] A. De Marco, Two-step metal affinity purification of double-tagged (NusA-His 6) fusion proteins, Nat. Protoc. 1 (2006) 1538

[27] J. Wang, X. Pan, P. Tien, et al., High level expression of 5-helix protein in HIV gp41 heptad repeat regions and its virus fusion-inhibiting activity, Chin. J. Biotechnol. 25 (2009) 435.

[28] D.M. Jameson, J.A. Ross, Fluorescence polarization/anisotropy in diagnostics and imaging, Chem. Rev. 110 (2010) 2685.

[29] M. Irving, Steady-state polarization from cylindrically symmetric fluorophores undergoing rapid restricted motion, Biophys. J. 1996 (1830) 70.

[30] X. Huang, Fluorescence polarization competition assay: the range of resolvable inhibitor potency is limited by the affinity of the fluorescent ligand, J. Biomol. Screen. 8 (2003) 34.

[31] Z. Gao, O.G. Ovchinnikova, B.-S. Huang, et al., High-throughput "FP-tag" assay for the identification of glycosyltransferase inhibitors, J. Am. Chem. Soc. 141 (2019) 2201.

[32] X. Yin, J. Li, S. Chen, et al., An Economical High-Throughput "FP-Tag" Assay for Screening Glycosyltransferase Inhibitors, ChemBioChem 22 (2020) 1391. 